PAPER

\title{
Chronic inflammatory demyelinating polyneuropathy: decreased claudin-5 and relocated ZO-1
}

\author{
T Kanda, Y Numata, H Mizusawa
}

See end of article for authors' affiliations

......................

Correspondence to: Dr T Kanda, Department of Neurology and

Neurological Science,

Tokyo Medical and Dental

University Graduate

School, 1-5-45 Yushima,

Bunkyo-ku, Tokyo

113-8519, Japan;

t-kanda.nuro@tmd.ac.jp

Received 11 August 2003

In revised form

29 September 2003

Accepted

30 September 2003
J Neurol Neurosurg Psychiatry 2004;75:765-769. doi: 10.1136/jnnp.2003.025692

Objectives: To clarify the dynamics of molecules composing the blood-nerve barrier (BNB) in inflammatory neuropathies.

Methods: The expression of four tight junction (TJ) proteins - claudin-1, claudin-5, occludin, and ZO-1 was analysed immunohistochemically in sural nerve biopsy specimens obtained from patients with chronic inflammatory demyelinating polyradiculoneuropathy (CIDP).

Results: Claudin-1 was detected only in perineurial cells, whereas claudin-5 was present in endothelial cells, irrespective of vessel location or size. Occludin and ZO- 1 were found in perineurial cells, in addition to some epineurial and endoneurial endothelial cells. In CIDP, percentages of endoneurial small vessels immunoreactive for claudin-5 were significantly decreased, as were ZO-1 immunoreactive endoneurial small vessels, with staining localised to interfaces between cells. Claudin-1 and occludin immunoreactivity did not differ appreciably between the neuropathies examined.

Conclusions: The downregulation of claudin-5 and altered localisation of ZO-1 seen in CIDP specimens may indicate that BNB derangement occurs in inflammatory neuropathies. Further investigation of TJ molecules may suggest new treatments based on properties of the BNB.
$\mathrm{E}$ ndothelial cells in the adult central nervous system (CNS) and also in the peripheral nervous system (PNS) are coupled by tight junctions (TJ) that resemble those of epithelial barriers ${ }^{12}$ and show extremely low permeability. Breakdown of these TJs in the blood-nerve barrier (BNB) may allow antimyelin antibodies and various inflammatory cytokines to enter peripheral nerve tissues, exacerbating peripheral nerve injury in inflammatory neuropathies, including Guillain-Barré syndrome ${ }^{3}$ and chronic inflammatory demyelinating polyneuropathy (CIDP). The development of new therapeutic strategies to restore BNB function based on the properties of TJs has long has been awaited. Understanding of the molecular basis of TJs has been increasing since 1993, when occludin, a protein with four transmembrane domains, was discovered. ${ }^{4}$ Occludin has also been detected in endothelial cells forming the blood-brain barrier (BBB). ${ }^{5}$ Occludin deficient cells have been found to form morphologically normal TJs. ${ }^{6}$ However, a new family of TJ proteins, claudins, has received attention more recently. Claudins also have four transmembrane domains, but do not show homology to occludin. ${ }^{78}$ Among claudin family members, claudin-1, claudin-5, and claudin- 11 have been demonstrated in the brain. ${ }^{78}$ Because claudin- 11 is expressed only in oligodendrocytes, ${ }^{9}$ and presumably is not related to endothelial barriers, we focused on claudin- 1 and claudin- 5 as molecules of interest concerning the barrier system in both the CNS and PNS. In our present study, using immunohistochemistry, we analysed the expression of four representative TJ proteins-claudin-1, claudin-5, occludin, and ZO-1in the human PNS using sural nerve biopsy specimens. We examined the expression of these molecules in PNS disorders in which BNB breakdown is believed to have an important pathogenetic role.

\section{MATERIALS AND METHODS Patients}

Sural nerve biopsy specimens obtained from 25 patients including 10 from patients with CIDP (eight men and two women; age range, 13-64 years; mean, 44.3) were studied. Informed consent was obtained from each patient. The study complied with the ethical guidelines of Tokyo Medical and Dental University. Diagnoses were based on detailed clinical and electrophysiological investigations of the patients, in addition to the pathological examination of sural nerve specimens, including toluidine blue stained semithin sections and teased fibre preparations. CIDP was diagnosed according to standard criteria, ${ }^{10}$ and all 10 patients were categorised as "definite" CIDP. Table 1 lists the clinical features and immunostaining results in the patients with CIDP. Other nerve biopsy specimens studied as disease controls included those obtained from six patients with vasculitic neuropathy associated with the Churg-Strauss syndrome (three men and three women; age range, 44-67 years; mean, 57.2); six with hereditary neuropathy (three men and three women; age range, 17-61 years; mean, 45.5; five with type I CharcotMarie tooth disease and one with hereditary sensory and autonomic neuropathy type II); and four with nutritional neuropathy resulting from vitamin $\mathrm{B}_{1}$ deficiency (three men and one woman; age range, 23-62 years; mean, 45.8). Specimens were snap frozen and stored at $-80^{\circ} \mathrm{C}$ until use.

\section{Immunohistochemical techniques}

Rabbit polyclonal antibodies against human claudin-1, claudin-5, occludin, and ZO-1 were purchased from Zymed (South San Francisco, California, USA). Serial transverse sections $(10 \mu \mathrm{m}$ thick) were cut from specimens on a cryostat, fixed in acetone at $4^{\circ} \mathrm{C}$ for five minutes, and then exposed to $0.03 \% \mathrm{H}_{2} \mathrm{O}_{2} /$ methanol for 10 minutes at room temperature. Sections were then preincubated in phosphate buffered saline (PBS) supplemented with 10\% normal goat serum for three hours before incubating overnight with

Abbreviations: $B B B$, blood-brain barrier; $B M E C$, brain microvascular endothelial cell; BNB, blood-nerve barrier; CNS, central nervous system; CIDP, chronic inflammatory demyelinating polyneuropathy; PBS, phosphate buffered saline; PNS, peripheral nervous system; TJ, tight junction; VEGF, vascular endothelial growth factor 
Table 1 Clinical features and immunostaining results in 10 patients with chronic inflammatory demyelinating polyradiculoneuropathy

\begin{tabular}{|c|c|c|c|c|c|c|c|c|c|}
\hline Patient & Age/sex & $\begin{array}{l}\text { Biopsy } \\
\text { (months) }\end{array}$ & $\begin{array}{l}\text { CSF protein } \\
(\mathrm{mg} / \mathrm{l})\end{array}$ & Symptoms & Course & $\begin{array}{l}\text { Nerve conduction } \\
\text { findings }\end{array}$ & $\begin{array}{l}\text { Antiganglioside } \\
\text { antibodies }\end{array}$ & $\begin{array}{l}\text { Anti-C5+ } \\
\text { microvessels (\%) }\end{array}$ & $\begin{array}{l}\text { ZO-1+ vessels } \\
\text { positive at endothelial } \\
\text { cell interfaces }(\%)\end{array}$ \\
\hline 1 & $13 M$ & 10 & 1170 & $\mathrm{Mo}>\mathrm{Se}$ & Progr & $D$ & - & 27.4 & 57.1 \\
\hline 2 & $24 \mathrm{M}$ & 6 & 960 & $\mathrm{Mo}=\mathrm{Se}$ & Progr & D & - & 70.9 & 11.1 \\
\hline 3 & $31 \mathrm{M}$ & 3 & 1670 & $\mathrm{Mo}=\mathrm{Se}$ & Progr & D & - & 38.4 & 40.0 \\
\hline 4 & $32 \mathrm{~F}$ & 4 & 880 & $\mathrm{Mo}=\mathrm{Se}$ & Progr & $D+A$ & GMI (lgM) & 69.4 & 78.1 \\
\hline 5 & $41 \mathrm{M}$ & 5 & 680 & $\mathrm{Mo}=\mathrm{Se}$ & RR & D & GMI, GDIb, SGPG & 72.6 & 85.7 \\
\hline 6 & $55 \mathrm{~F}$ & 2 & 1140 & $\mathrm{Mo}=\mathrm{Se}$ & Progr & D & - & 93.2 & $\dagger$ \\
\hline 7 & $58 \mathrm{M}$ & 6 & 1100 & $\mathrm{Mo}<\mathrm{Se}$ & Progr & D & SGLPG & 52.2 & 41.1 \\
\hline 8 & $62 \mathrm{M}$ & 3 & 1610 & $\mathrm{Mo}<\mathrm{Se}$ & RR & D & - & 94.5 & 13.3 \\
\hline 9 & $63 \mathrm{M}$ & $72+$ & 9520 & $\mathrm{Mo}=\mathrm{Se}$ & Progr & $\mathrm{D}$ & - & 63.2 & 33.3 \\
\hline 10 & $64 \mathrm{M}$ & 2 & 1850 & $\mathrm{Mo}=\mathrm{Se}$ & $R R$ & D & SGLPG & 24.3 & 55.6 \\
\hline
\end{tabular}

*Percentage of ZO-1 immunoreactive endoneurial vessels showing immunoreactivity at endothelial cell interfaces; †ZO-1 immunoreactivity was too faint for the percentage of vessels showing immunoreactivity at endothelial cell interfaces to be evaluated.

Age, age at the time of biopsy; Biopsy, months from disease onset to biopsy; CSF, cerebrospinal fluid; C5, claudin-5; Mo, motor symptoms; Se, sensory symptoms; Course, clinical course until biopsy; Progr, progressive; RR, relapsing and remitting; D, demyelinating; A, axonal; SGPG, sulfoglucuronosyl paragloboside; SGLPG, sulfoglucuronosyl lactosaminyl paragloboside.

primary antibody diluted in PBS. Anti-claudin-5 and antioccludin antibodies were used at a 1/400 dilution, whereas others were used at a $1 / 800$ dilution. Sections were then rinsed with PBS three times before incubation for one hour with peroxidase conjugated secondary antibody (Nichirei, Tokyo, Japan). The reaction product indicating immunoreactivity in sections was developed with diaminobenzidine.

\section{Analysis of claudin-5 positive microvasculature}

We analysed endoneurial microvessels less than $30 \mu \mathrm{m}$ in diameter, which included capillaries and some precapillary arterioles and postcapillary venules. Vessels apparently surrounded by perineurial cell layers were excluded. Because microvessels in the endoneurium were difficult to count accurately in cryostat sections, whether haematoxylin and eosin stained or immunostained using endothelial markers, such as anti-von Willebrand antigen, we first counted these vessels using toluidine blue stained, plastic embedded semithin sections to evaluate the density of endoneurial microvessels $/ \mathrm{mm}^{2}$. The percentage of claudin5 -positive endoneurial microvessels was calculated as the density of anti-claudin-5 immunoreactive microvessels in cryostat sections $\times 100$ /overall density of microvessels in toluidine blue stained sections. If the first density exceeded the last, the percentage of claudin-5 immunoreactive microvessels was considered to be $100 \%$. More than $0.5 \mathrm{~mm}^{2}$ surface area of endoneurial space was evaluated in each specimen.

\section{Analysis of staining pattern by anti-ZO-1 antibody}

The percentage of endoneurial microvessels showing ZO-1 immunoreactivity localised at interfaces between adjoining endothelial cells was evaluated. Microvessels showing two or more immunoreactive lines across the endothelial cell layer, or two or more immunoreactive dots located at the luminal side of endothelial cells (fig l) were judged to have ZO-1 localised at these endothelial cell borders. The percentage of ZO-1 immunoreactive cells with this localisation pattern was calculated as the number of endoneurial microvessels showing linear or punctate ZO-1 immunoreactivity at borders between endothelial cells $\times 100$ /overall number of $\mathrm{ZO}-1$ immunoreactive endoneurial microvessels.

\section{RESULTS}

Claudin-1 immunoreactivity was evenly present in the perineurial cell layers, with no clear difference in immunoreactivity between the inner and outer layers. No specimen showed staining of endothelial cells (figs 2A and 3). No
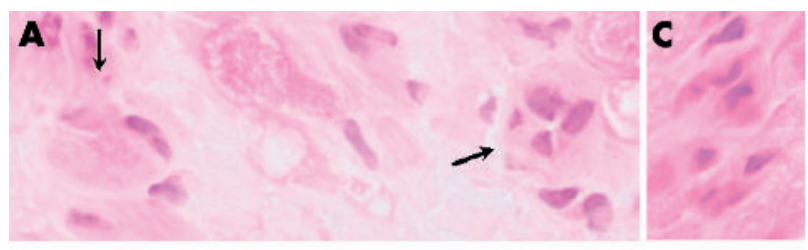

B
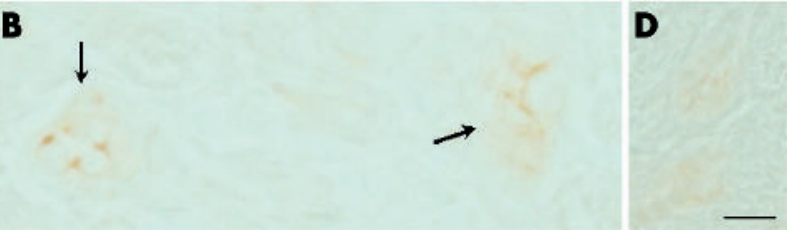

Figure 1 Immunostaining with anti-ZO-1 antibody ( $B$ and $D$ ) and corresponding serial sections ( $A$ and $C$; haematoxylin and eosin staining) in sural nerves from a 67 year old woman with Churg-Strauss syndrome (A and B) and a 58 year old man with chronic inflammatory demyelinating polyneuropathy (CIPD) (C and D). ZO-1 immunoreactivity in endoneurial microvessels was localised to the interfaces between endothelial cells (arrows), especially at the luminal surface (note the punctate staining of the microvessel on the left in B). In CIDP, endothelial immunoreactivity was diffuse and weak (D); most strong staining localised to the intercellular borders had disappeared. Bar, $10 \mu \mathrm{m}$.

difference in claudin-1 immunoreactivity was noted between CIDP and the other disease groups (fig 3 ).

The polyclonal anti-occludin antibody stained the perineurial cell layers. In addition, the luminal aspect of some epineurial vessels and a small proportion of endoneurial capillaries adjacent to the perineurium was also immunoreactive (fig $2 \mathrm{~B}$ ). Endoneurial microvessels situated near the centre of the endoneurial space that were not neighbouring perineurial cell layers were not stained. No appreciable difference in immunoreactivity was noted between CIDP and the other disease groups.

Anti-ZO-1 immunoreactivity was localised to the perineurial cell layers, in addition to some endothelial cells lining epineurial vessels and the endoneurium (fig 2C). Although no detectable background staining was seen with the antioccludin, anti-claudin- 1 , and anti-claudin-5 antibodies, faint background immunoreactivity was occasionally seen in the endoneurium with the anti-ZO-1 antibody. Therefore, the exact percentage of stained endoneurial microvessels was difficult to determine. Instead, we calculated the percentage of endoneurial microvessels showing immunoreactivity localised to the junctions between endothelial cells (fig 1). In CIDP specimens, the percentage of endoneurial microvessels 

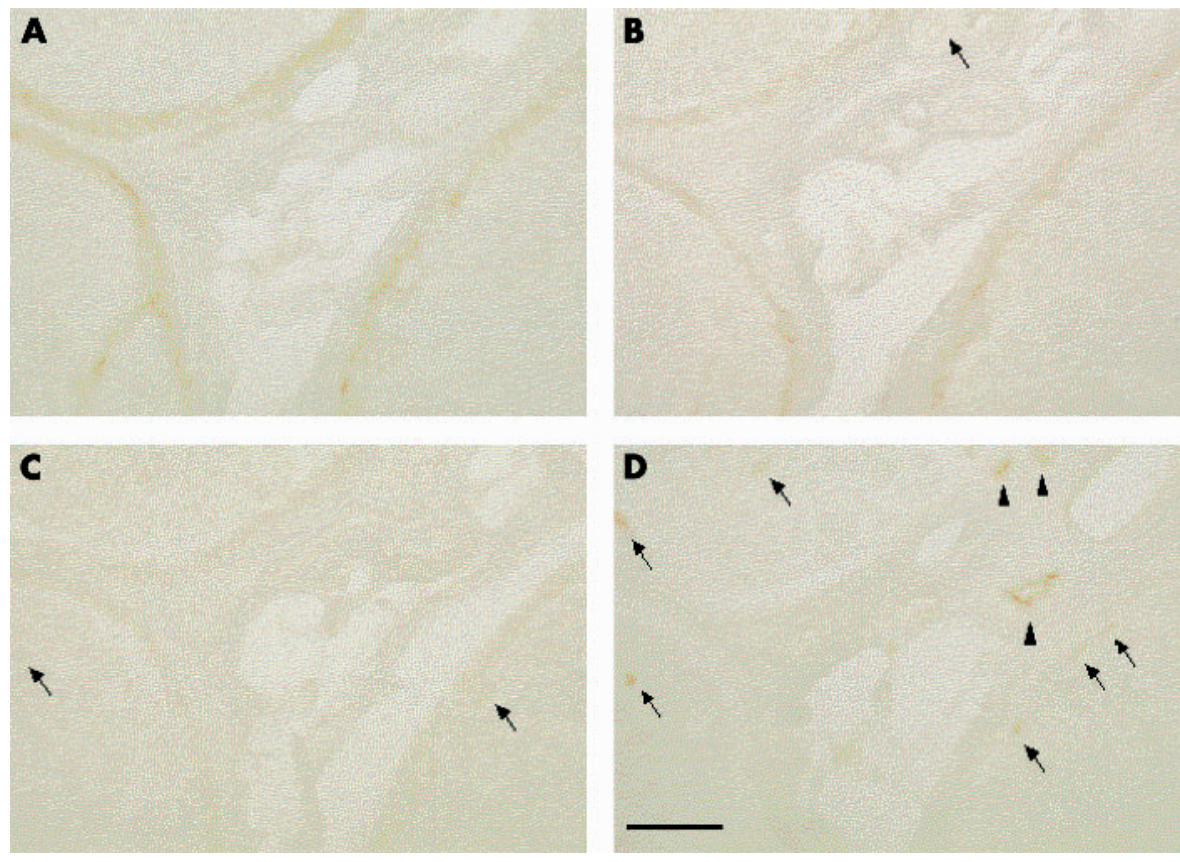

Figure 2 Immunostaining in serial sections obtained from a 64 year old man with Churg-Strauss syndrome with (A) anti-claudin-1, (B) anti-occludin, (C) anti-ZO-1, and (D) anti-claudin-5 antibodies, showing a normal pattern of staining in the human peripheral nervous system. (A) Anti-claudin-1 antibody stained perineurial cell layers exclusively, with no immunoreactivity against endothelial cells. (B) Antioccludin immunoreactivity was noted in perineurial cell layers. In addition, faint staining was seen in the endothelial cell layers of epineurial vessels (arrow). (C) The anti-ZO-1 antibody reacted faintly with epineurial cell layers and some of the endothelial cells in the endoneurium (arrows). (D) Unlike the other three antibodies, anti-claudin-5 antibody stained endothelial cells exclusively, irrespective of their vessel size or location. Endothelial cells in the epineurium (arrowheads) and in the endoneurium (arrows) were immunoreactive. Bar, $100 \mu \mathrm{m}$. with such anti-ZO-1 immunoreactivity localisation was significantly lower (fig 4).

Claudin-5 was detected exclusively in endothelial cells, irrespective of the location or size of the vessel in the control specimen (figs 2D and 5). Unlike anti-ZO-1 immunostaining, immunoreactivity was not detected at endothelial cell interfaces. In CIDP, the percentage of anti-claudin-5 immunoreactive microvessels in the endoneurium was significantly decreased compared with non-inflammatory neuropathies (figs 5 and 6). No apparent correlation was noted between the loss of claudin-5 immunoreactivity and endoneurial/ subperineurial oedema

\section{DISCUSSION}

In inflammatory neuropathies including CIDP, increases in cytokines such as interleukin $1 \beta,{ }^{11}{ }^{12}$ tumour necrosis factor $\alpha_{1}{ }^{11}{ }^{12}$ and vascular endothelial growth factor (VEGF) ${ }^{13}$ are
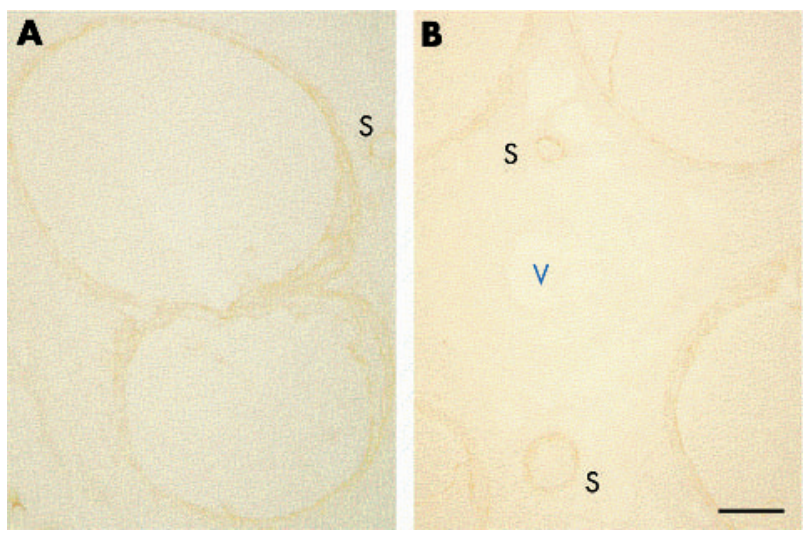

Figure 3 Claudin-1 immunostaining in a control specimen from (A) a 67 year old woman with Churg-Strauss syndrome (CSS) and (B) a specimen from a 55 year old woman with chronic inflammatory demyelinating polyneuropathy (CIDP). Claudin-1 immunoreactivity was detected exclusively in the perineurial cell layers, and no endothelial staining was noted in the endoneurium or epineurium (V). Sections from CIDP and CSS were stained equally. $V$ designates a medium sized epineurial vessel and $\mathrm{S}$ designates small sized nerve fascicles. Bar, $100 \mu \mathrm{m}$. believed to contribute to pathogenesis through modulation of the BNB. Among these, VEGF acts as a particularly potent disrupter of the BNB in various inflammatory neuropathies. ${ }^{14}$ This increase in vascular permeability occurs through the binding of VEGF to its tyrosine kinase-type receptors, flt- 1 and flt- $\mathrm{k},{ }^{15}$ resulting in a decrease in TJ proteins, including occludin and vascular endothelial cadherin, and subsequent disorganisation of interendothelial cell junctions. ${ }^{16}$ Thus, study of the molecular dynamics of TJ proteins in human inflammatory neuropathies may improve understanding of BNB derangement and prompt the development of new therapeutic approaches in these disorders. Recent experiments have indicated that the establishment of TJ strands depends on claudin family proteins. For example, the transfection of claudin- 1 and claudin-2 into fibroblasts induces the formation of TJ strands. ${ }^{17}$ Claudin- 11 knockout

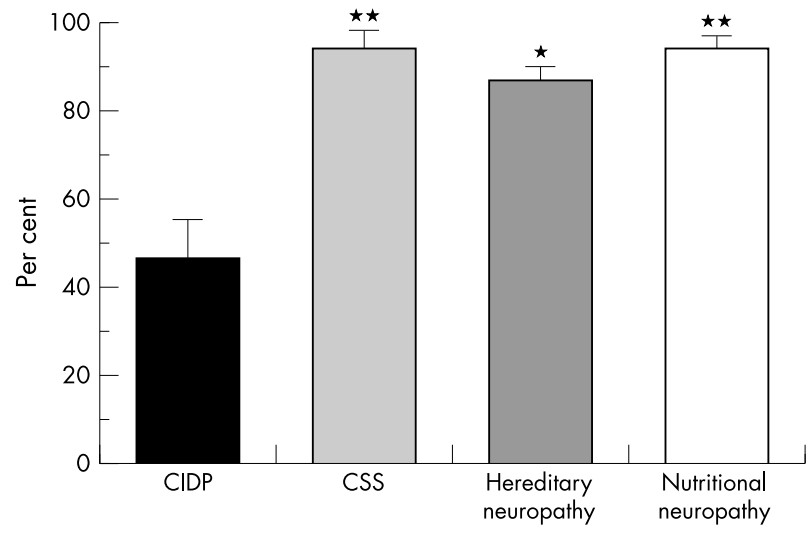

Figure 4 Mean percentage of all ZO-1 immunoreactive endoneurial microvessels showing localisation of ZO-1 immunoreactivity to the interfaces between endothelial cells in specimens with chronic inflammatory demyelinating polyneuropathy (CIDP) (mean, 46.1\%; SEM , 8.6\%), Churg-Strauss syndrome (CSS) (mean, 93.8\%; SEM, 3.7\%), hereditary neuropathy (mean, $86.3 \% ;$ SEM, $3.1 \%$ ), and nutritional neuropathy (mean, 94.0\%; SEM, 2.1\%). CIDP specimens showed a significantly lower percentage of endoneurial microvessels with this localisation of ZO- 1 immunoreactivity. ${ }^{*} p<0.005$ v CIDP; ${ }^{* *} \mathrm{p}<0.002 \vee$ CIDP. Bars indicate SEM. 


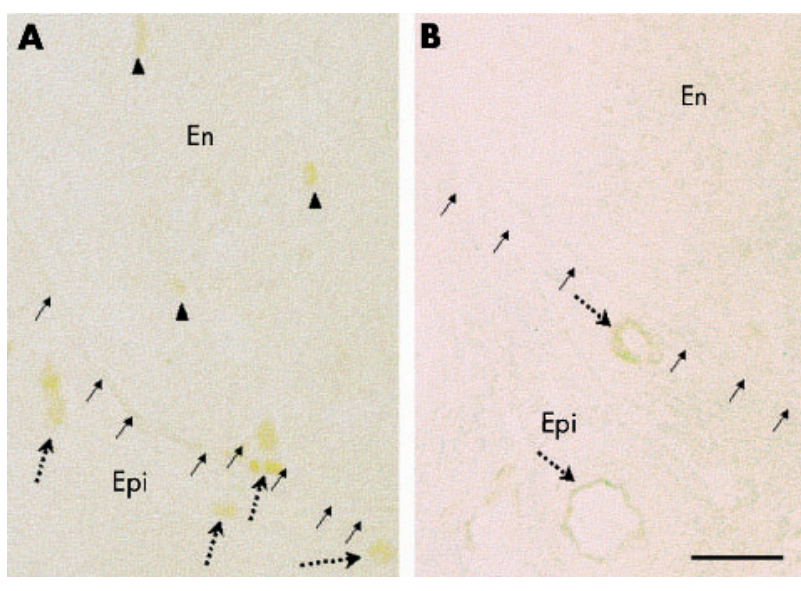

Figure 5 Claudin-5 immunostaining in (A) a control specimen from a 67 year old woman with Churg-Strauss syndrome and (B) a 31 year old man with chronic inflammatory demyelinating polyneuropathy (CIDP). The small arrows indicate the outer aspect of the endoneurium. In the control (A), claudin-5 immunoreactivity was detected exclusively in endothelial cells, irrespective of vessel size or location. Dotted arrows indicate immunoreactive microvessels in the epineurium and arrowheads indicate those in the endoneurium. In CIDP (B), although medium sized vessels in the epineurium were well stained (dotted arrows), immunoreactivity in the endoneurium was severely decreased. En, endoneurium; Epi, epineurium. Bar, $50 \mu \mathrm{m}$.

mice show absence of TJ strands in the myelin sheets of oligodendrocytes and Sertoli cells, ${ }^{18}$ whereas claudin-16 knockout mice demonstrate abnormal paracellular passage of $\mathrm{Mg}^{2+}$ ions. ${ }^{19}$ Accordingly, we first focused on the expression of two claudin family members, claudin-1 and claudin-5, in sural nerve biopsy specimens.

Claudin-5, which localises exclusively to borders between adjacent endothelial cells, ${ }^{20}$ is considered to be important in the control of vascular permeability. Recently Nitta et al reported the selective leakage of small molecules $(<800 \mathrm{Da})$ across the BBB in claudin-5 deficient mice. ${ }^{21}$ This suggests that claudin-5, although ubiquitously present in all endothelial cells, plays a special role in the barrier mechanism of the nervous system by preventing the entrance of such small molecules. In their article, no information was given about the BNB, but free entrance of small molecules through claudin-5 deficient endothelial junctions may also occur in the PNS. Hence, the loss of claudin-5 in the endoneurial microvessels in patients with CIDP may enhance the leakage of small molecules into the endoneurial space, and may result in changes to the endoneurial constituents that are unfavourable to Schwann cells and axons, eventually leading to the worsening of neuropathy. However, we found no correlation between the loss of claudin-5 immunoreactivity and the presence of endoneurial/subperineurial oedema; in addition, $\mathrm{Cld} 5^{-/-}$mice do not show vasogenic oedema. This might be explained by the fact that even in claudin-5 deficiency most serum proteins (molecular mass $>800 \mathrm{Da}$ ) cannot extravasate. ${ }^{21}$ Endoneurial/subperineurial oedema, a relatively common finding in sural nerve specimens from patients with CIDP, ${ }^{22}$ may be elicited by factors other than a decrease in claudin-5 expression.

The disagreement between our BNB findings and previous observations concerning the $\mathrm{BBB}^{20}$ is somewhat difficult to account for; one explanation is that downregulation of claudin-5 in endoneurial microvessels in CIDP might be a BNB specific phenomenon, reflecting differences in anatomic structure and cell populations (for example, the absence of astrocytes). Of 10 CIDP specimens, two (from patients 6 and 8) showed normal anti-claudin-5 immunoreactivity in

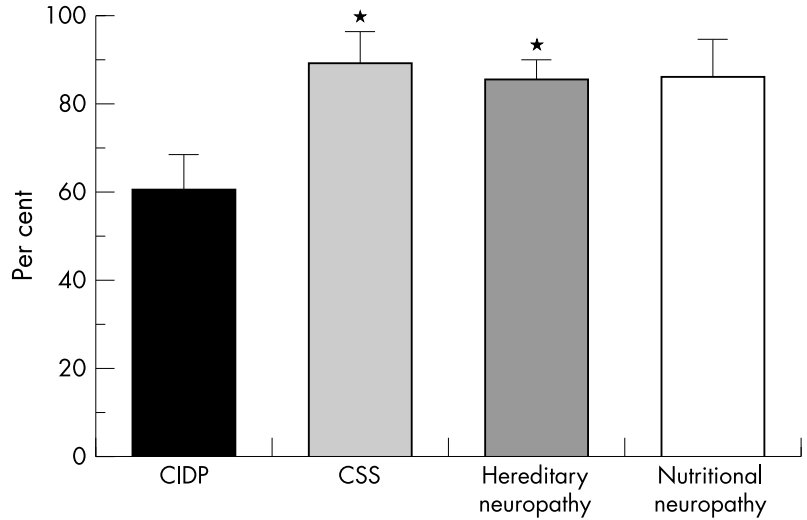

Figure 6 Mean percentage of endoneurial microvessels immunoreactive with anti-claudin-5 antibody in specimens with chronic inflammatory demyelinating polyneuropathy (CIDP) (mean, 60.6\%; SEM, 7.8\%), Churg-Strauss syndrome (CSS) (mean, 89.5\%; SEM, 6.5\%), hereditary neuropathy (mean, $85.6 \%$; SEM, $4.0 \%$ ), and nutritional neuropathy (mean, 86.2\%; SEM, 8.4\%). Staining with the anti-claudin-5 antibody in the endothelial cells of the endoneurium was significantly decreased in CIDP specimens compared with CSS and hereditary neuropathy. ${ }^{*} p<0.05 \vee$ CIDP. Bars indicate SEM.

endoneurial microvessels; this could reflect a non-uniform or multifocal, rather than diffuse, distribution of demyelinative foci in this disorder. ${ }^{23}$ However, these two specimens showed extremely faint ZO-1 immunoreactivity (patient 6) or a very low percentage of endoneurial vessels showing ZO- 1 immunoreactivity at intercellular interfaces (patient 8). Thus, changes of expression of claudin- 5 and ZO- 1 are not always in parallel with CIDP, suggesting functional differences between these two TJ proteins.

Although claudin-1 is the only member of the claudin family (except for claudin-5) that might be expected to be present in endothelial cells, we found no staining for claudin- 1 in the endothelial cells of the PNS. Instead, the anticlaudin-1 antibody exclusively stained the perineurial cell layers. Because the BNB includes the endoneurial microvasculature and the innermost layer of the perineurium, ${ }^{24}{ }^{25}$ anti-claudin-1 immunoreactivity might be taken to represent the latter. However, the uniform staining of all perineurial cell layers that was seen does not correspond well to the site of the BNB. In addition, claudin-1 immunoreactivity was almost as abundant in CIDP as in control specimens. Therefore, claudin-1 is not a marker of BNB integrity.

In our present study, we detected occludin immunoreactivity in endothelial cells of some epineurial vessels, a small percentage of endoneurial capillaries adjoining perineurial cell layers, and perineurial cells. However, we saw no appreciable differences in occludin immunoreactivity in the perineurial cell layer and in the endothelial cells between the various disorders. Occludin may not be essential for TJ formation, ${ }^{6}$ but it has been reported to be abundant in relation to the endothelial cells of the brain, although it is undetectable in non-neural tissues. ${ }^{56}$ VEGF treatment of brain microvascular endothelial cell (BMEC) monolayer cultures decreased detectable occludin and disrupted its continuous pericellular distribution. ${ }^{27}$ Although these reports suggest that occludin may play some part in the maintenance of BNB integrity, our results indicated that occludin does not change appreciably in inflammatory neuropathies.

$\mathrm{ZO}-\mathrm{l}$, a $220 \mathrm{kDa}$ TJ phosphoprotein, is a member of the membrane associated guanylate kinases localised to intercellular contacts. ${ }^{28}$ ZO- 1 binds various proteins, including claudins and occludin, and may act as a molecular scaffold bringing these TJ constituents together. ${ }^{29}$ Therefore, ZO- 1 is expected to be a key molecule in the control of BNB integrity, 
despite some previous conclusions that the expression and localisation of ZO-1 do not correlate with the physiological efficiency of paracellular barrier function. ${ }^{30}$ In CIDP specimens, we found no significant decrease in ZO-l immunoreactive endoneurial microvessels, although we noted a change in the staining pattern. This corresponds well with a recent observation that VEGF, known to open the BBB and $\mathrm{BNB},{ }^{14}$ caused a loss of ZO-1 from endothelial cell junctions and changed the staining pattern at the cell boundary without decreasing ZO-1 content in cultured bovine BMEC. ${ }^{27}$ We suspect that the change in the localisation of ZO-1 in CIDP specimens was an effect of various cytokines, including VEGF, that are upregulated in inflammatory neuropathies such as CIDP. The contribution of other inflammatory cytokines in addition to VEGF requires future investigation.

\section{Authors' affiliations}

T Kanda, Y Numata, H Mizusawa, Department of Neurology and Neurological Science, Tokyo Medical and Dental University Graduate School, 1-5-45 Yushima, Bunkyo-ku, Tokyo 113-8519, Japan

Competing interest: none declared

\section{REFERENCES}

1 Reese TJ, Karnovsky MJ. Fine structural localization of a blood-brain barrier to exogenous peroxidases. J Cell Biol 1967;34:207-17.

2 Rubin LL. Endothelial cells: adhesion and tight junction. Curr Opin Cell Biol 1992;4:830-3

3 Kanda T, Yamawaki M, Mizusawa H. Sera from Guillain-Barré patients enhance leakage in blood-nerve barrier model. Neurology 2003;60:301-6.

4 Furuse $M$, Hirase $T$, Itoh $M$, et al. Occludin: a novel integral membrane protein localizing at tight junctions. J Cell Biol 1993;123:1777-88.

5 Hirase T, Staddon JM, Saitou M, et al. Occludin as a possible determinant of tight junction permeability in endothelial cells. J Cell Sci 1997; 1 10:1603-13.

6 Saitou M, Fujimoto K, Doi Y, et al. Occludin-deficient embryonic stem cells can differentiate into polarized epithelial cells bearing tight junctions. J Cell Biol 1998; 141:397-408

7 Furuse M, Fujita K, Hiiragi T, et al. Claudin-1 and -2: novel integral membrane proteins localizing at tight junctions with no sequence similarity to occludin. $J$ Cell Biol 1998;141:1539-50.

8 Morita K, Furuse M, Fujimoto K, et al. Claudin mulitigene family encoding four-transmembrane domain protein components of tight junction strands. Proc Natl Acad Sci U S A 1999;96:511-16.

9 Morita K, Sasaki H, Fujimoto K, et al. Claudin-11/OSP-based tight junctions of myelin sheaths in brain and Sertoli cells in testis. J Cell Biol 1999;145:579-88.
10 Research criteria for diagnosis of chronic inflammatory demyelinating polyneuropathy (CIDP). Report from an ad hoc subcommittee of the American Academy of Neurology AIDS Task Force. Neurology 1991;32:958-64.

11 Sharief MK, Ingram DA, Swash M. Circulating tumor necrosis factor-alpha correlates with electrodiagnostic abnormalities in Guillain-Barré syndrome. Ann Neurol 1997;42:68-73.

12 Zhu J, Bai XF, Mix E, et al. Experimental allergic neuritis: cytolysin mRNA expression is upregulated in lymph node cells during convalescence. J Neuroimmunol 1997;78:108-16.

13 Watanabe O, Arimura K, Kitajima I, et al. Greatly raised vascular endothelial growth factor (VEGF) in POEMS syndrome. Lancet 1996;347:702.

14 Kanda T, Iwasaki T, Yamawaki M, et al. Anti-GM1 antibody facilitates leakage in an in vitro blood-nerve barrier model. Neurology 2000;55:585-7.

15 Gale NW, Yancopoulos GD. Growth factors acting via endothelial cellspecific receptor tyrosine kinases: VEGFs, angiopoietins, and ephrins in vascular development. Genes Dev 1999;13:1055-66.

16 Kevil CG, Payne DK, Mire E, et al. Vascular permeability factor/vascular endothelial cell growth factor-mediated permeability occurs through disorganization of endothelial junctional proteins. J Biol Chem 1998;273: 15099-103.

17 Tsukita S, Furuse M. Pores in the wall: claudins constitute tight junction strands containing aqueous pores. J Cell Biol 2000;149:13-16.

18 Gow A, Southwood CM, Li JS, et al. CNS myelin and Sertoli cell tight junction strands are absent in Osp/claudin-11 null mice. Cell 1999;99:649-59.

19 Simon DB, Lu Y, Choate KA, et al. Paracellin-1, a renal tight junction protein required for paracellular $\mathrm{Mg}^{2+}$ resorption. Science 1999;285:103-6.

20 Morita K, Sasaki H, Furuse M, et al. Endothelial claudin: claudin-5/TMVCF constitutes tight junction strands in endothelial cells. J Cell Biol 1999; 147:185-94.

21 Nitta T, Hata M, Gotoh S, et al. Size-selective loosening of the blood-brain barrier in claudin-5 deficient mice. J Cell Biol 2003;161:653-60.

22 Kanda T, Yamawaki M, Iwasaki T, et al. Glycosphingolipid antibodies and blood-nerve barrier in autoimmune demyelinative neuropathy. Neurology 2000;54:1459-64.

23 Lewis RA, Sumner AJ. Electrophysiologic features of inherited demyelinating neuropathies: a reappraisal. Ann N Y Acad Sci 1999;883:321-5.

24 Bell MA, Weddell AGM. A descriptive study of the blood vessels of the sciatic nerve in the rat, man, and other mammals. Brain 1984;107:871-98.

25 Latker $\mathrm{CH}$, Wadhwani KC, Baldo A, et al. Blood-nerve barrier in the frog during Wallerian degeneration: are axons necessary for maintenance of barrier function? J Comp Neurol 1991;309:650-64.

26 Saitou $M$, Ando-Akatsuka $Y$, Itoh $M$, et al. Mammalian occludin in epithelial cells: its expression and subcellular distribution. Eur J Cell Biol 1997:73:222-31.

27 Wang W, Dentler WL, Borchardt RT. VEGF increases BMEC monolayer permeability by affecting occludin expression and tight junction assembly. Am J Physiol $2001 ; 280: \mathrm{H} 434-40$.

28 Mitic LL, Anderson JM. Molecular architecture of tight junctions. Annu Rev Physiol 1998:60:121-42.

29 Zahraoui A, Louvard D, Galli T. Tight junction, a platform for trafficking and signaling protein complexes. J Cell Biol 2000;151:F31-6.

30 Stevenson BR, Anderson JM, Goodenough DA, et al. Tight junction structure and ZO-1 content are identical in two strains of Madin-Darby canine kidney cells which differ in transepithelial resistance. J Cell Biol 1988;107:2401-8. 